\title{
Metastatic hepatocellular carcinoma of the esophagus: an unusual cause of upper gastro- intestinal bleeding
}
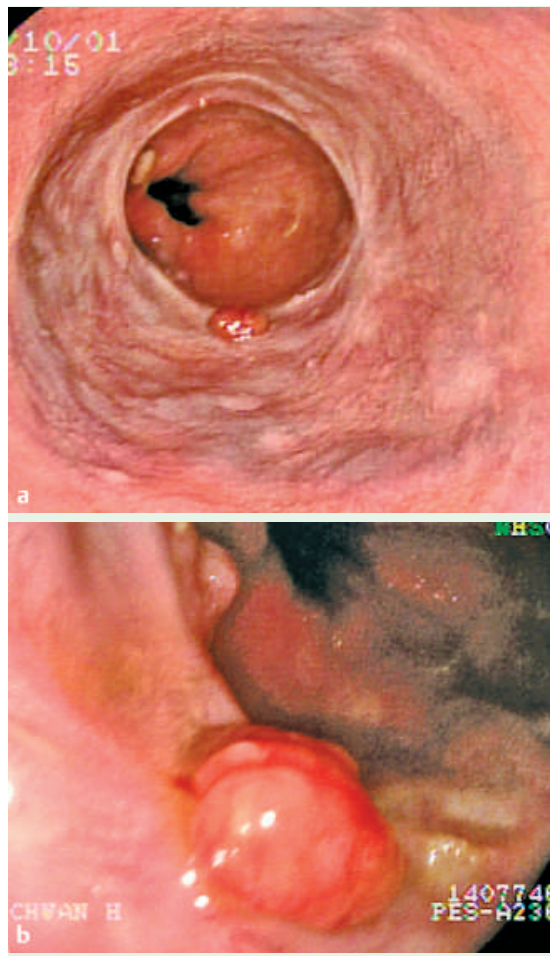

Fig. 1 a Endoscopic view showing small esophageal varices without red marks and one polypoid lesion near the esophagogastric junction. b Closer endoscopic view showing surface oozing of the polypoid lesion.

Hepatocellular carcinoma (HCC) is a hypervascular tumor that frequently metastasizes through systemic vessels or the portal system. Lung, adrenal gland and bone are the common sites of distant metastasis of HCC [1]. On the other hand, the incidence of gastrointestinal tract metastasis of HCC is typically low, with the stomach, duodenum and colon being the most commonly involved organs [2]. Esophageal metastasis is especially rare in patients with HCC [1]. The major symptoms of metastatic HCC of the esophagus include dysphagia and gastrointestinal bleeding [3 - 5]. Endoscopically, metastatic HCC of the esophagus may present as a submucosal tumor or a polypoid mass [3-5]. We report here a case of metastatic HCC of the esophagus, which presented as an oozing polypoid lesion at upper gastrointestinal endoscopy.

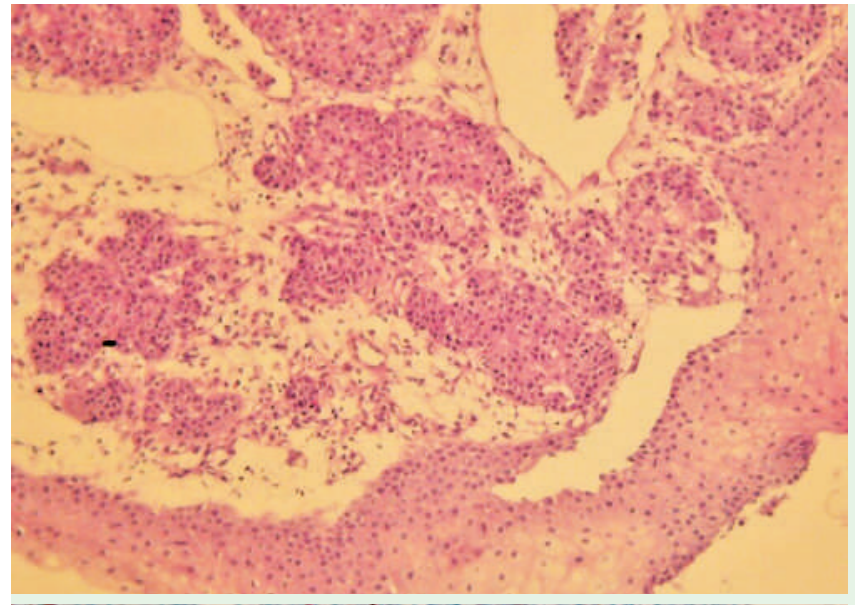

Fig. 2 a Photomicrograph showing nests of tumor cells in a trabecular arrangement in the subepithelial region (H\&E, original magnification $\times 100)$.

b Photomicrograph showing brown cytoplasmic staining, indicating that the tumor cells are positive for monoclonal antibody hepatocyte paraffin 1 (original magnification $\times 100)$.

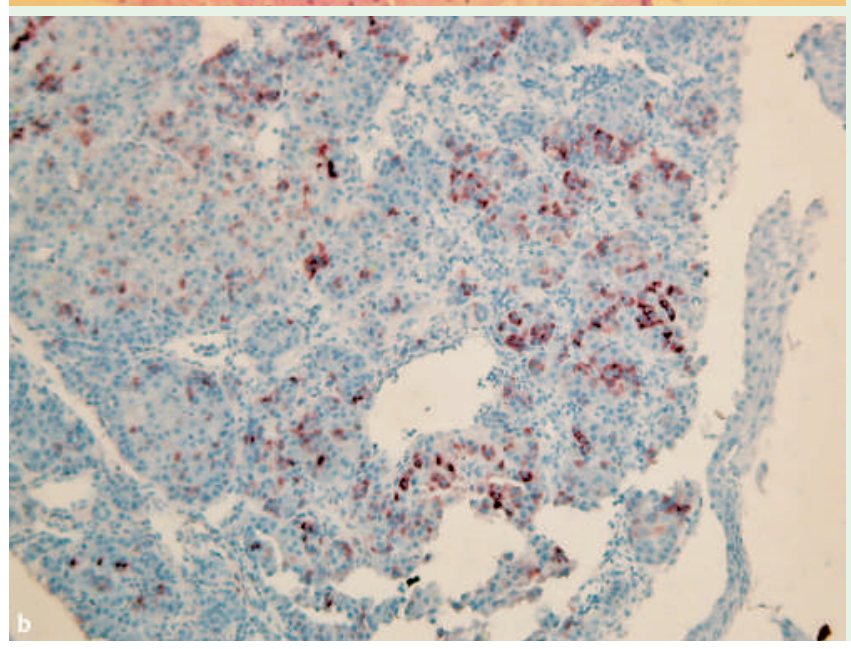

A 53-year-old man presented at our institution with a 1-day history of melena. His past medical history was notable for hepatitis-B-related cirrhosis and hepatocellular carcinoma. Physical examination revealed anemia, jaundice and a slightly distended abdomen with ascites. Laboratory data included a hematocrit of $22.9 \%$ (normal $42 \%-52 \%$ ), a mean corpuscular volume of $100 / \mathrm{fl}$ (normal $80-94 / \mathrm{fl}$ ), a total bilirubin level of $4.9 \mathrm{mg} / \mathrm{dl}$ (normal $0.2-1.2 \mathrm{mg} / \mathrm{dl}$ ), and an albumin level of $2.2 \mathrm{~g} / \mathrm{dl}$ (normal $3.8-5.3 \mathrm{~g} / \mathrm{dl}$ ). Prothrombin time was $16.4 \mathrm{~s}$ (normal 10-14 s) and $\alpha$-fetoprotein was $17036 \mathrm{ng} / \mathrm{ml}$ (normal $<13.4 \mathrm{ng} / \mathrm{ml}$ ). Previous computed tomography of the abdomen demonstrated a spreading-type HCC in the left lobe of liver with portal vein tumor thrombi. Urgent upper gastrointestinal endoscopy showed small esophageal varices without red marks, and a $0.6-\mathrm{cm}$ polypoid lesion with an oozing surface near the esophagogastric junction ( $\bullet$ Fig. 1). No ulcer or any other bleeding focus was found in the stomach or duodenum. We concluded that the polypoid lesion had bled and caused melena. Biopsy specimens were taken and argon plasma coagulation was performed for hemostasis. The histopathological assessment of the biopsy specimens demonstrated nests of tumors cells in a trabecular arrangement in the subepithelial region ( $\bullet$ Fig. 2 a). Immunohistochemical staining revealed positivity for monoclonal antibody hepatocyte paraffin 1 (Hep Par 1), confirming the liver as the primary origin of metastasis ( Fig. 2 b). A diagnosis of metastatic HCC was made. The patient died of pro- 
gressive hepatic failure 1 month later, and autopsy was not permitted.

Endoscopy_UCTN_Code_CCL_1AB_2AC_3AB

S.-L. Yan, Y.-H. Hung, T.-H. Yang

Division of Gastroenterology, Department of Internal Medicine, Chang Bing ShowChwan Memorial Hospital, Taiwan, Republic of China

\section{References}

1 Nakashima T, Okuda K, Kojiro M et al. Pathology of hepatocellular carcinoma in Japan. 232 Consecutive cases autopsied in ten years. Cancer 1983; 51: 863-877

2 Chen LT, Chen CY, Jan CM et al. Gastrointestinal tract involvement in hepatocellular carcinoma: clinical, radiological and endoscopic studies. Endoscopy 1990; 22: $118-$ 123

3 Kume K, Murata I, Yoshikawa I et al. Polypoid metastatic hepatocellular carcinoma of the esophagus occurring after endoscopic variceal band ligation. Endoscopy 2000; 32 : 419-421

4 Sohara N, Takagi H, Yamada T et al. Esophageal metastasis of hepatocellular carcinoma. Gastrointest Endosc 2000; 51: 739-741

5 Cho A, Ryu M, Yoshinaga Yet al. Hepatocellular carcinoma with unusual metastasis to the esophagus. Hepatogastroenterology 2003; 50: $1143-1145$
Bibliography

DOI $10.1055 / \mathrm{s}-2007-966480$

Endoscopy 2007; 39: 257-258

(c) Georg Thieme Verlag KG Stuttgart · New York . ISSN 0013-726X

\section{Corresponding author}

\section{Y.-H.Hung, MD}

Division of Gastroenterology,

Department of Internal Medicine

Chang Bing Show-Chwan Memorial Hospital

No. 6, Lugong Road

Lugang Township

Changhua County 505

Taiwan, Republic of China

Fax: +886-4-7233190

yslendo2@yahoo.com 